\title{
Zur Theorie der Oberflächenzustände ${ }^{1}$
}

\author{
Von Hermann Statz \\ Aus dem Institut für theoretische und angewandte Physik der Technischen Hochschule Stuttgart \\ (Z. Naturforschg. 5 a, 534-544 [1950]; eingegangen am 21. Oktober 1950)
}

\begin{abstract}
Beim eindimensionalen Gitter wird systematisch zwischen zwei Arten von Oberflächenzuständen (O.Z.) unterschieden, je nachdem sie aus einem oder aus zwei Bändern entstammen. Die O.Z. erster Art werden aus einem neuen, einfachen Gesichtspunkt heraus, ohne jede Rechnung, anschaulich verständlich gemacht. Bei den O.Z. zweiter Art wird nicht mehr das Energiespektrum, wie das bisher üblich war, als Funktion der Gitterkonstanten $a$, sondern als Funktion eines Parameters $\alpha$, wie er in der Theorie der Hillschen Differentialgleichungen verwendet wird, aufgetragen. Indem man auch negative Werte von $\alpha$ heranzieht, lassen sich die bisher anschaulich nicht zu erfassenden O.Z. und der ganze Mechanismus des Austretens aus den Bändern durch ein einfaches Abzählverfahren mit Hilfe von Überlegungen, wie sie bei den O.Z. erster Art angestellt wurden, direkt verstehen. In diesem Schema zeigt sich dann auch in besonders deutlicher Weise, wie die Arbeit von $\mathrm{Maue}{ }^{4}$ mit der von $\mathrm{Shockle} \mathrm{y}{ }^{8}$ zusammenhängt. Unsere Betrachtungsweise gestattet noch einige wichtige Schlüsse über die Energiespektren der Elektronen in Kristallen. Es läßt sich die Erwartung aussprechen, daß für „richtig zwischen zwei Atcmen abgeschnittene“ Kristalle im ersten verbotenen Gebiet, wegen des Fehlens von Kreuzungsstellen, keine O.Z. zweiter Art auftreten.
\end{abstract}

Bei mehrdimensionalen Kristallen wird darauf hingewiesen, daß die von anderer Seite versuchte Übertragung des Ergebnisses bezüglich der O.Z. zweiter Art vom eindimensionalen auf mehrdimensicnale Gitter ein ganz irreales Potential zugrundelegt. Damit wird die auch schon vom Experiment widerlegte Behauptung, daß der Diamant eine Oberflächenleitfähigkeit zeigen scllte, entkräftet. Nur im Falle fast freier Elektronen ist die eindimensionale Rechnung übertragbar. Es läßt sich aber dann meist für mehrere Brillouinsche Zonen zeigen, daß für sie keine O.Z. und damit keine leitenden Oberflächenbänder auftreten können. Unter gewissen Annahmen ist es erlaubt, diesen Zonen die interessierenden Valenzbänder zuzuordnen. Um die Kreuzungsstellen von Bändern mehrdimensionaler Probleme weiter zu untersuchen, wird zum erstenmal die Zellenmethode zur Berechnung der O.Z. benützt. An dem durchgerechneten Gitter, das als zweidimensionales Analogon zum Diamanten angesprochen werden kann, treten die O.Z. an der Kreuzungsstelle nur aus dem oberen leeren Band aus, führen also zu keiner Oberflächenleitfähigkeit. Die Zuverlässigkeit der Zellenmethode läßt sich hierbei noch nicht völlig sicher beurteilen.

Un $m$ die in der Elektronentheorie der Festkörper auftretenden Gleichungen lösen zu können, muß man stets einschneidende Vereinfachungen machen. So hat man sich bis jetzt fast immer darauf beschränkt, das im Kristall vorliegende Vielkörperproblem auf ein Einkörperproblem zu reduzieren und dabei die Wechselwirkung der Elektronen mit den Kernen und der Elektronen untereinander, soweit dies überhaupt möglich ist, in ein Pauschalpotential des Einelektronenproblems zu stecken. Dieses Vorgehen hat sich, trotz seiner starken Vereinfachung, in vielen Fällen

1 „Oberflächenzustände“ im Text durch O.Z. abgekürzt.

2 I. T a m m, Physik. Z. Sowjetunion 1, 733 [1932].

3 S. Ri j a n ow, Z. Physik 89, 806 [1934].

4 A. M a u e, Z. Physik 94, 717 [1935].

5 E. T. G o odw in, Proc. Cambridge philos. Soc. 35 , 205 [1939].

6 E. T. G o o dw in, Prcc. Cambridge philos. Soc. 35, 221 [1939].

7 E. T. G o o dwin, Proc. Cambridge philos. Soc. 35 , 232 [1939]. gut bewährt. Nun haben im Rahmen des Einelektronenproblems verschiedene Verf. ${ }^{2-8}$ darauf hingewiesen, daß bei der Lösung der Schrödinger-Gleichung für den endlichen Kristall unter gewissen Annahmen Eigenfunktionen auftreten können, die von der Oberfläche weg ins Innere des Kristalles exponentiell gedämpft sind. Diese sog. O.Z. entsprechen Elektronen, die eine überwiegende Aufenthaltswahrscheinlichkeit in der Randschicht haben und daher zu gewissen Oberflächeneffekten Anlaß geben können. Willkommen waren diese Zustände bei der Erklärung von Erscheinungen an Halbleitern ${ }^{9-13}$. Dagegen sollte

8 W. Shockley, Physic. Rev. 56, 317 [1939].

9 J. Bardeen u. W. Brattain, Physic. Rev. 75 , 1208 [1949].

10 S. B e nzer, Progress Report, Contract No. W-36039-SC-32 020, Purdue University (1. Sept.-30. Nov. 1946).

11 S. B e n z e r, Physic. Rev. 71, 141 [1947].

12 W. Shockley u. G. Pearson, Physic. Rev. 74, 232 [1948].

13 W. B r a tta in, Physic. Rev. 72, 345 [1947]. 
ihre Existenz zu einer bei Diamant nicht beobachteten Oberflächenleitfähigkeit führen. Dieser Widerspruch mag es u. a. rechtfertigen, das Thema erneut aufzugreifen. Es wird im folgenden ohne Rücksicht auf mögliche Gitterverzerrungen ${ }^{14}$ am Rand bearbeitet.

Der unendliche Kristall besitzt, schon eindimensional, das bekannte Bänderspektrum von Energiezuständen. Schneidet man aus ihm den endlichen Kristall heraus, so treten in diesem Spektrum zwei wesentliche Veränderungen ein: Erstens lösen sich die vorher kontinuierlichen Bänder in mehr oder weniger dichte Folgen diskreter Niveaus auf, - bei hinreichender Größe des Kristalls bedeutet dies praktisch kaum einen Unterschied - Zweitens scheidet sich aus der Mannigfaltigkeit der Bänderzustände das abgesonderte Spektrum der oben erwähnten O.Z. aus. Wir unterscheiden im folgenden beim eindimensionalen, endlichen Kristall zwischen zwei Arten von O.Z., je nachdem, ob sie aus einem oder zwei Bändern stammen. In unserer noch näher zu erläuternden Sprechweise würden sich die Arbeiten ${ }^{2,6}$ mit dem O.Z. erster Art und die Arbeiten ${ }^{4,8}$ mit denen zweiter Art befassen. Wir haben damit gleichzeitig eine Einteilung getroffen, die den verschiedenartigen $\mathrm{Be}$ setzungsverhältnissen der O.Z. Rechnung trägt: Am absoluten Nullpunkt sind die O.Z. erster Art zusammen mit dem Band, aus dem sie entstammen, entweder vollständig besetzt oder vollständig unbesetzt. Die O.Z. zweiter Art dagegen sind dann halb besetzt, wenn sie aus einem unteren vollen und einem oberen leeren Band ausgetreten sind. Bei mehrdimensionalen Kristallen geben dann solche halbgefüllten "Oberflächenbänder“ zu einer Oberflächenleitfähigkeit Anlaß. Durch bisher noch nicht angestellte Überlegungen sind wir in der Lage, diese beiden Arten von O.Z. aus gemeinsamen Gesichtspunkten heraus zu verstehen und einige weitergehende Folgerungen
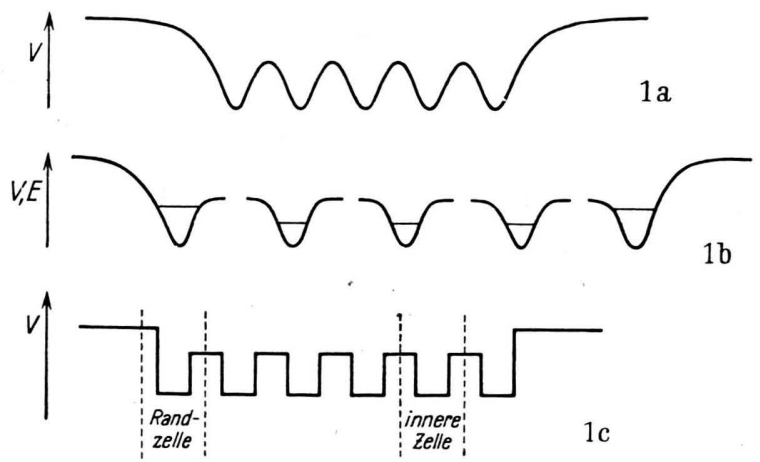

Abb. 1. Schematische Potentialbilder zur Erklärung der Oberflächenzustände erster Art. zu ziehen. Das Analogon zu den eindimensionalen O.Z. erster Art tritt auch bei mehrdimensionalen Kristallen auf; wir behandeln sie deshalb im Zusammenhang mit diesen. Dagegen erfordern die O.Z. zweiter Art im mehrdimensionalen Fall eine besondere Betrachtungsweise. Mit Hilfe der Zellenmethode finden wir ein Ergebnis, das kein Analogon im eindimensionalen Gitter hat und welches das Versagen der bisherigen Argumentation für das Auftreten von O.Z. beim Diamanten direkt zeigt.

\section{Eindimensionale Kristalle}

Oberflächenzustände erster Art

Die Abb. $1 \mathrm{a}$ und $1 \mathrm{c}$ sind grobe, schematische Bilder des Potentialverlaufes in Kristallen. Die Mulden im Potential entsprechen den Orten der Atomrümpfe. Das wesentlich Gemeinsame ist, daß die „Randelementarzellen“ von den inneren Zellen verschieden sind. Dieser abgeänderte Potentialverlauf in den äußersten Zellen kann etwa durch das Fehlen der zum Potential beitragenden Nachbaratome begründet werden. Ohne nun die Rechnung der Verfasser ${ }^{2, ~}{ }^{\circ}$ durchzuführen, können wir durch eine einfache Überlegung die O.Z. mit Hilfe der Abb. 1 b verstehen. Man denkt sich den Kristall durch Zusammenrücken weit entfernter Potentialtöpfe allmählich entstanden. Wir fragen nach dem Energiespektrum der Quantenzustände ihrer Elektronen. Einfachheitshalber ist nur ein Eigenwert in die Potentialtöpfe eingezeichnet. Hierbei sind die Quantenenergien in den inneren Potentialtöpfen einander gleich, in den Randtöpfen wegen des abweichenden Potentials etwas höher. Beim Zusammenrücken der Töpfe kombinieren nun die Eigenfunktionen der inneren Zellen miteinander, d. h. jede Eigenfunktion pflanzt sich etwa gleich stark in die anderen inneren Zellen fort. Es entstehen bekanntlich hierdurch neue "Gitterzustände“, die sich durch den ganzen Kristall hindurchziehen. Die Eigenwerte der inneren Töpfe spalten hierbei etwas auf und bilden ein „Band“ im Energiespektrum. Die äußeren Töpfe kombinieren jedoch, wenn ihr Eigenwert hinreichend von dem der inneren Zellen verschieden ist, fast nicht mit diesen. Die Eigenfunktion der Randzellen klingt in den Nachbarzellen exponentiell ab. Die beiden Eigenwerte der Randzellen liegen über dem Band der inneren Zellen. Der eben beschriebene Vorgang ist durchaus vergleichbar mit klassischen Resonanzerscheinungen,

14 J. C. S l a te r, Physic. Rev. 76, 1592 [1949]. 
wenn man die Frequenz des klassischen Problems in Analogie setzt zur Energie des quantenmechanischen Problems.

Eine ganz entsprechende Begründung läßt sich auch für die O.Z. erster Art in dreidimensionalen Gittern geben. Die Anzahl der O.Z. ist einfach gleich der Anzahl der deformierten Potentialtöpfe, d. h. gleich der Anzahl der Oberflächenatome. Wenn man die Abweichung des Potentials in den Randtöpfen langsam auflöst, so verschwinden die O.Z. und finden sich als normale Zustände im Band wieder, d. h. die O.Z. „entstammen“ diesem Band. Vollständigkeitshalber sei hier noch hinzugefügt, daß die obenstehende Betrachtung nur dann sinnvoll ist, wenn die Energie niedriger als die Höhe der Potentialberge im Kristall ist. Es ist dann vielleicht eine von S hock le y ${ }^{8}$ angedeutete Störungsrechnung berechtigt.

\section{Oberflächenzustände zweiter Art}

Indem man ein eindimensionales Gitter durch Zusammenrücken gleicher Töpfe entstanden denkt und hierbei das Energiespektrum als Funktion der Gitterkonstanten $a$ aufträgt, erhält man nach Shockley das in Abb. 2 dargestellte qualitative Bild. Der mit $a$ sich gänzlich ändernde Charakter des Energiespektrums rührt daher, daß beim Zusammenrücken die Potentialberge zwischen den Mulden immer mehr abgetragen werden. Hierbei beeinflussen sich die zuerst ungestörten Eigenfunktionen der einzelnen Zellen mehr und mehr, und die Bänder werden immer breiter, bis sich schließlich die Grenzkurven kreuzen. Man sieht, daß gerade beim Kreuzen aus dem oberen und unteren Band je ein O.Z. ins verbotene Gebiet austritt. Wie später noch näher begründet werden soll,

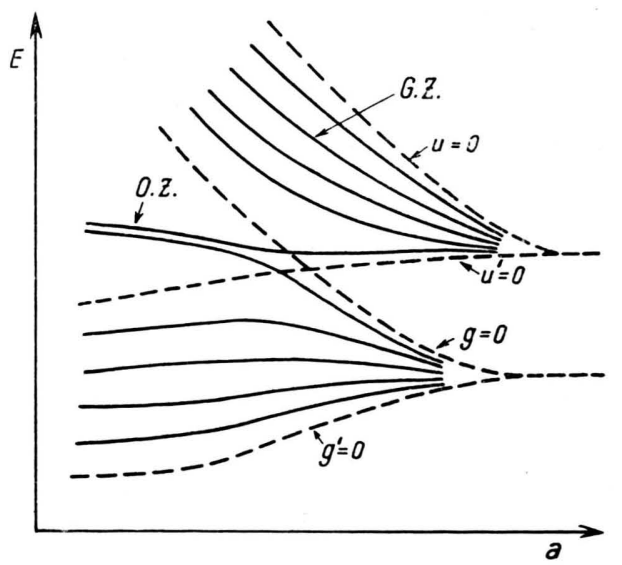

Abb. 2. Energiespektrum eines aus nur gleichen Zellen bestehenden eindimensionalen, endlichen Kristalles als Funktion der Gitterkonstanten $a$ (nach Shockley). G.Z. = Gitterzustände. entstammen diese O.Z. im Gegensatz zu den oben beschriebenen aus zwei verschiedenen Bändern!

Wir knüpfen an die obigen Überlegungen hinsichtlich der Verkleinerung der Gitterkonstanten an und kommen dabei zu einer für das Folgende grundlegenden Betrachtung: Bei einer etwa gleichbleibenden Höhe der Niveaus sinken die Potentiaiberge durch das Abtragen immer tiefer und werden schließlich niedriger als die Energien der Zustände. Man gelangt dabei von dem Bereich der ,gebundenen“ $\mathrm{zu}$ dem der „fast freien“ Elektronen. Ähnlich liegen die Verhältnisse, wenn wir in der Schrödinger-Gleichung für den eindimensionalen Kristall

$$
\frac{d^{2} \psi}{d x^{2}}+\frac{2 m}{\hbar^{2}}(E-a V(\tilde{x})) \psi=0
$$

den Parameter $\alpha$ variieren. Für große Werte von $\alpha$ haben wir „lokalisierte“ Zustände, für kleine Werte von $\alpha$ fast freie Elektronen. Man kann daher mit einem gewissen Recht den Parameter $\alpha$ in Analogie zur Gitterkonstanten setzen. Wir sehen dabei bewußt von der mit $a$ immer kleiner werdenden Periode des Potentials als für unsere Überlegungen unwesentlich ab. Den Nullpunkt von $E$ und $V$ wollen wir so wählen, daß in (1) und weiter unten auch im endlichen Kristall das gemittelte Potential $\bar{V}=0$ ist. Die oben beschriebene Umdeutung besitzt den Vorteil, daß man in (1) die sogenannte Hillsche Differentialgleichung vor sich hat, deren Grenzkurven der Bänder in der $E, \alpha$-Ebene für bestimmte Formen des periodischen Potentials $V(\tilde{x})$ schon bekannt sind ${ }^{15}$. Das Energiespektrum für ein Cosinuspotential ist in Abb. 3 als Funktion von $\alpha$ aufgezeichnet.

Indem man den Kristall endlich macht, werden von den unendlich vielen Lösungen der Gl. (1) diejenigen ausgelesen, die den zusätzlichen Bedingungen des Anschlusses an eine gedämpfte Vakuumwelle genügen. Das Spektrum wird diskret. Das Spektrum rechts von der Achse $\alpha=0$ entspricht durchaus dem Spektrum der Abb. 2, wobei großen Werten von $a$ große Werte von $a$ und kleinen Werten von $a$ kleine Werte von $a$ zuzuordnen sind. Die Anzahl der Potentialtöpfe haben wir für $\alpha>0$, des guten Überblickes wegen, zu $n=4$ gewählt, obwohl ein realer Kristall ungleich mehr Töpfe hat und unsere folgende Betrachtung nur für große Zahlen von $n$ streng gültig ist. Bekanntlich gibt es damit auch in jedem Band vier diskrete Zustände. In unserer Abb. 3 haben wir noch das Spektrum für Werte von $\alpha<0$ gezeichnet,

15 M. J. O. S trutt, Ergebn. Math. 1, 24, 40 [1932]. 
obwohl dieses Gebiet in der ursprünglichen Abb. 2 sinngemäß nicht auftritt. Diese unphysikalisch erscheinende Erweiterung vermittelt uns jedoch ein gutes Verständnis für den in Abb. 2 merkwürdig anmutenden Mechanismus des Austretens der Zustände. In der Abb. 3 ist außerdem noch jeweils das Potential einerseits für große positive $a$ und andererseits für große negative $a$ gezeichnet. Für $\alpha=0$ schneiden sich die Grenzkurven in jedem zweiten verbotenen Gebiet, wir auch in jedem Band einen Zustand weniger. Dafür sind zwei halbe Randzellen aufgetreten, deren zugehörige zwei O.Z. in jedem zweiten verbotenen Gebiet liegen, - wie es auch nur sein darf, wenn in der Abb. 3 und weiter unten auch in der Abb. 4 die Anzahl der Niveaus in einem Band mit der der normalen Potentialtöpfe übereinstimmen soll. - Bemerkenswert ist, daß für den Spezialfall des reinen Cosinuspotentials unsere Überlegungen auch dann
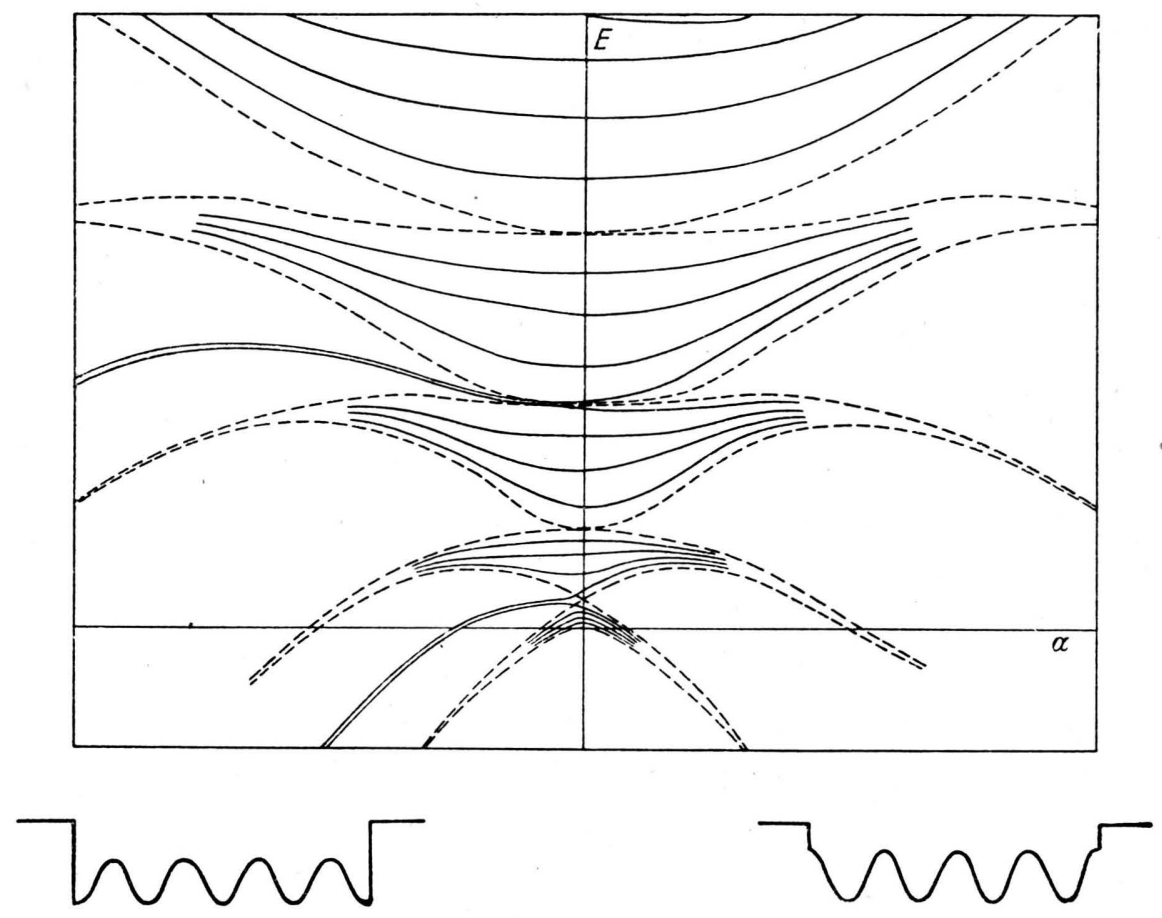

Abb. 3. Energiespektrum eines eindimensionalen, endlichen Kristalles als Funktion des Parameters $\alpha$. Im unteren rechten und linken Teil der Abb. ist das zugrunde gelegte cosinusförmige Potential für große positive und negative Werte von $\alpha$ dargestellt.

bzw., genauer ausgedrückt, berühren sich von erster, dritter, fünfter usw. Ordnung. Zeichnet man nun die einzelnen Zustände nach dem Vorbild von Abb. 2 auch für negative Werte von $\alpha$ ein, so treten in jedem zweiten verbotenen Gebiet bei $\alpha=0$ O.Z. aus. Man erkennt für große negative $\alpha$ sofort ihre Bedeutung: Es sind die Eigenfunktionen, die zu den halben Potentialtöpfen am Rande gehören. Man sieht, daß ihre Eigenwertdichte, da sie nur in jedem zweiten verbotenen Gebiet auftreten, etwa halb so groß ist wie die der normalen Töpfe. Im übrigen ist die Abb. 3 ganz zwangsläufig zu verstehen. Wie aus dem darunter gezeichneten Potentialverlauf zu ersehen ist, hat die Anzahl der normalen Potentialtöpfe für negative $\alpha$ um eins abgenommen. Entsprechend finden noch gelten, wenn $a V_{\max } \ll E$, obwohl dies nicht ohne weiteres zu erwarten ist (vgl. den Schluß des Abschnittes über O.Z. erster Art).

Einen tieferen Einblick erhalten wir noch, wenn wir das etwas kompliziertere Rechteckpotential betrachten (Abb. 4). Wir können hier wieder genau die obigen Abzählungen der Zustände für $\alpha V_{\max } \gg E$ durchführen. Geändert hat sich allerdings, wie auch zu erwarten war, der Charakter des Spektrums für Werte von $\alpha V_{\max } \ll E$. Nur in diesem Grenzgebiet gilt die von $\mathrm{Maue}^{4}$ durchgeführte Rechnung. Es wird dort in der bekannten Brillouinschen Weise das Potential in eine Cosinusreihe entwickelt, wobei als Koordinatenanfangspunkt die Abschneidestelle des Kristalles gewählt ist. Bekanntlich geht dann bei der 
Berechnung der Zustände in der Umgebung des ersten verbotenen Gebietes nur der erste Fourier-Koeffizient in die Rechnung ein, beim zweiten nur der zweite usw. Dementsprechend ist auch die Breite des $l$-ten verbotenen Gebietes nur vom $l$-ten Fourier-Koeffizienten abhängig und ergibt sich zu $2\left|\alpha V_{l}\right|$. Die Rechnung ergibt, daß in dieser Näherung im $l$-ten stelle gemäß des Potentialbildes in Abb. 4 für $\alpha>0$ : $V_{1}>0, V_{2}=0, V_{3}<0, V_{4}=0, V_{5}>0$, usw. Im ersten verbotenen Gebiet gibt es daher für $\alpha>0$ keine O.Z., dagegen aber für $\alpha<0$, weil sich dort das Vorzeichen von $\alpha V_{1}$ umgekehrt hat. Über das zweite verbotene Gebiet kann man wegen des Verschwindens des Koeffizienten in dieser Näherung
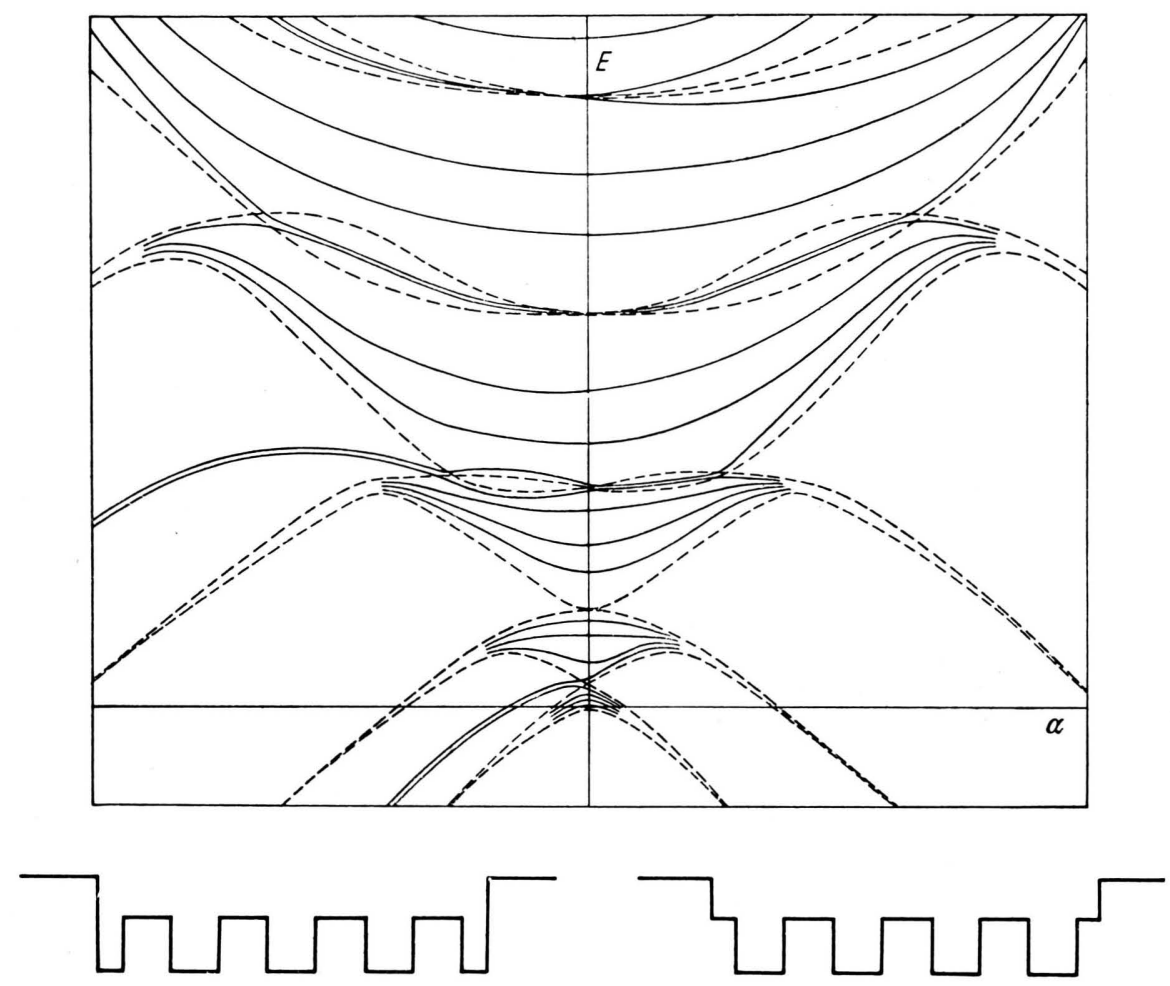

Abb. 4. Energiespektrum eines eindimensionalen, endlichen Kristalles als Funktion des Parameters $\alpha$. Im unteren rechten und linken Teil der Abb. ist das zugrunde gelegte rechteckförmige Potential für große positive und negative Werte von $\alpha$ dargestellt. Im Gegensatz zur Abb. 3 schneiden sich hier die Grenzkurven der Bänder auch außerhalb der Achse $a=0$.

verbotenen Gebiet O.Z. nur dann existieren, wenn der $l$-te Fourier-Koeffizient negativ ist. Dieses Ergebnis kann man als Spezialfall der Abb. 3 verstehen, wenn man beachtet, daß, wie oben schon bemerkt, in die Brillouinschen Rechnungen ein reines Cosinuspotential eingeht. Es entsprechen sich dann der allein vorhandene Koeffizient $V_{1}$ und das erste verbotene Gebiet der Abb. 3 einerseits und der Koeffizient $V_{l}$ und das $l$-te verbotene Gebiet der Rechnung andererseits. Aus der Abb. 3 aber entnehmen wir, daß nur für $\alpha<0$, d. h. $\alpha V_{1}<0$, O.Z. im ersten verbotenen Gebiet vorhanden sind. Durch diese Betrachtung wird uns das Auftreten dieser Art von O.Z. rechts von der Achse $\alpha=0$ in Abb. 4 verständlich. Für das Rechteckpotential ist bei der Entwicklung um die Abschneide- nichts aussagen. Im dritten verbotenen Gebiet sind dementsprechend nahe der Achse für $\alpha>0$ O.Z. vorhanden, für $\alpha<0$ dagegen nicht. Im vierten verbotenen Gebiet läßt sich wiederum unmittelbar nichts aussagen, usw. Andererseits kennen wir nun aus unseren früheren Betrachtungen das Verhalten für $\alpha V_{\text {max }} \geqslant E$. Wir können z. B. schließen, daß im dritten verbotenen Gebiet rechts von der Achse noch mindestens eine Kreuzungsstelle vorhanden sein muß, da für große positive $\alpha$ keine O.Z. vorkommen können. Entsprechend kann man auch für $\alpha<0$ schließen. Es müssen dort für große negative $\alpha$ O.Z. vorhanden sein und damit auch eine Kreuzungsstelle außerhalb der Achse für $\alpha<0$.

Einen Teil der oben beschriebenen Folgerungen 
kann man auch noch etwas anders gewinnen: Ėin aus nur gleichen Zellen bestehender Kristall ist auf beiden Enden jeweils am Rand einer Zelle abgeschnitten. Wie aus der Abb. 2 hervorgeht, sind hierbei die Grenzkurven im Energiespektrum durch $g=0, u=0$, $g^{\prime}=0$ und $u^{\prime}=0$ bezeichnet. Hierin bedeutet z. B. $g=0$, daß dié Grenzkurve gerade dieselbe Energie besitzt wie der bezüglich der Mitte einer Zelle gerade Anteil $g$ der Lösung der Schrödinger-Gleichung, der am Rande dieser Zelle einen Knoten, also $g=0$, hat. Bei $g^{\prime}=0$ hat die Ableitung dort einen Knoten. Für den ungeraden Anteil $u$ gilt entsprechend dasselbe. Der Grundgedanke ist nun, daß man in der Umgebung von $\alpha=0$ das Potential als Störung einführt und die Energie der Grenzkurven einfach als Störungsenergie der oben beschriebenen Funktionen berechnet. $\mathrm{Zu}$ diesem Zweck wird das Potential des Kristalles in eine Fourier-Reihe entwickelt. Als Nullpunkt wählt man den Rand des Kristalles, der mit dem Rand einer Zelle zusammenfällt. Hierbei ergibt sich, daß die Reihenfolge der Grenzkurven im $l$-ten verbotenen Gebiet vom Vorzeichen des $l$-ten FourierKoeffizienten abhängt. Wenn der Fourier-Koeffizient negativ ist, so ist die Reihenfolge der Grenzkurven entgegengesetzt derjenigen für große positive $\alpha$. Bemerkenswert ist, daß diese Reihenfolge sogar bis zur zweiten Ordnung in der Störungsenergie nur von dem einen Koeffizienten bestimmt wird. Mit Hilfe dieser Überlegung kann man z. B. in der Abb. 4 ebenso wie oben schließen, daß im dritten verbotenen Gebiet außerhalb der Achse $a=0$ noch Kreuzungsstellen vorhanden sein müssen.

Um Energiespektren von Kristallen analog zu den Abb. 3 und $4 \mathrm{zu}$ entwerfen, ist es, wie gezeigt, wesentlich, die Fourier-Entwicklung des Potentials bezüglich der Abschneidestelle des Kristalles zu kennen. Indem wir den Kristall, wie es physikalisch einzig sinnvoll ist, zwischen zwei Atomen durchschneiden, beschränken wir uns auf das Gebiet $\alpha>0$. Wir können dann für den ersten Fourier-Koeffizienten aussagen, daß er, unabhängig von der speziellen Form des Potentials, positiv ist. Dementsprechend treten auch nahe der Achse im ersten verbotenen Gebiet keine O.Z. auf. Aus der Kenntnis, daß für große a ebenfalls keine O.Z. vorhanden sind, kann man schließen, daß, vorsichtig ausgedrückt, keine ungerade Zahl von Kreuzungsstellen auftritt. Für das erste verbotene Gebiet kann man erwarten, daß man den Schluß verschärfen und sagen darf: Es gibt in diesem Gebiet wegen des Fehlens von Kreuzungsstellen keine O.Z. Für höhere verbotene Gebiete kann diese
Verschärfung nicht mehr zulässig sein. (So etwa beim fünften verbotenen Gebiet des Rechteckpotentials.)

Obwohl eindimensionale Kristalle nicht existieren, soll hier eine Bemerkung angeknüpft werden, die erst weiter unten bei den noch zu nennenden Erweiterungen auf mehrere Dimensionen wesentlich wird. Für die Valenzelektronen kann man manchmal die sehr nahe beieinander am Kern liegenden Knoten der Eigenfunktion vernachlässigen und als gute Näherung, vor allem in den äußeren Gebieten einer Elementarzelle, die Eigenfunktion durch eine ebene Welle annähern. Man vergleiche die in dem Artikel von $\mathrm{Slater}{ }^{16}$ gezeichneten $3 \mathrm{~s}-$ Funktionen von Natrium. Dies bedeutet aber nach dem „Oszillationstheorem“, daß man die Zählung der Bänder erniedrigt bzw., wenn man sämtliche kernnahen Knoten vernachlässigen kann, neu beginnt. Unter dieser Annahme gewinnt erst unsere obige Betrachtung an Bedeutung, weil wir damit mit dem ersten verbotenen Gebiet unserer Rechnung das vor allem interessierende verbotene Gebiet über dem Valenzband erfassen. Wir wollen hier aber nicht verschweigen, daß diese Betrachtung noch Schwierigkeiten der Zuordnung in sich birgt.

\section{Mehrdimensionale Kristalle}

Erweiterung der eindimensionalen Rechnung

Nur die unter der Einschränkung $\alpha V_{\max } \ll E$ gewonnenen Ergebnisse lassen sich unter derselben Bedingung auf mehrdimensionale Probleme übertragen. Legt man eine Oberfläche parallel zu einer Netzebenenschar, so kommt es in dieser Näherung wiederum nur darauf an, diejenigen Fourier-Koeffizienten zu kennen, die diese Netzebenenschar darstellen. Liegt die ideal zu denkende Oberfläche in der Mitte zwischen zwei Netzebenen (früher zwischen zwei Atomen), so kann man wieder, genau wie oben, schließen, daß bei einer Entwicklung mit dem Nullpunkt in der Oberfläche der erste Fourier-Koeffizient in der Darstellung dieser Netzebene positiv ist. In Abb. 5 ist für ein ebenes Rechteckgitter das BrillouinZonenschema gezeichnet. In diesem Schema kann man bekanntlich jeder dort gezeichneten Geraden ein Glied in der Fourier-Entwicklung des Potentials zuordnen. Es sind hier diejenigen Geraden, die in unserer Bezeichnung zu ersten Fourier-Koeffizienten gehören, ausgezogen, diejenigen, die zu höheren Koeffizienten gehören, gestrichelt. Man erkennt, daß in Abb. 5 beim

16 J. C. S l a t e r, Rev. mod. Physics 6, 247 [1934]. 

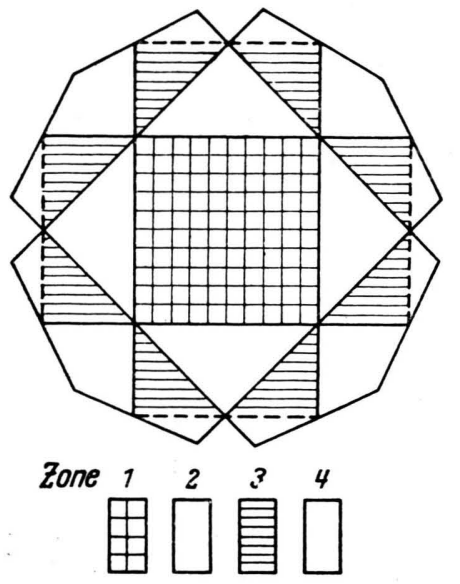

Abb. 5.

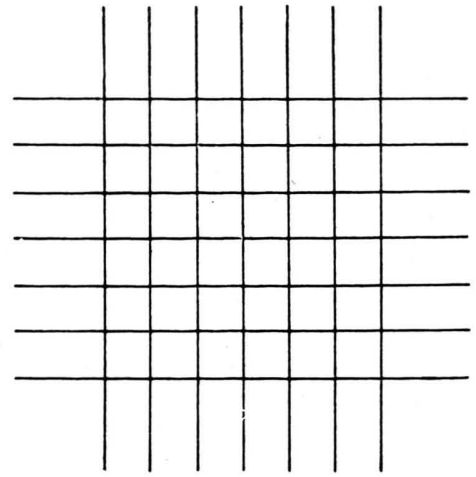

Abb. 6.

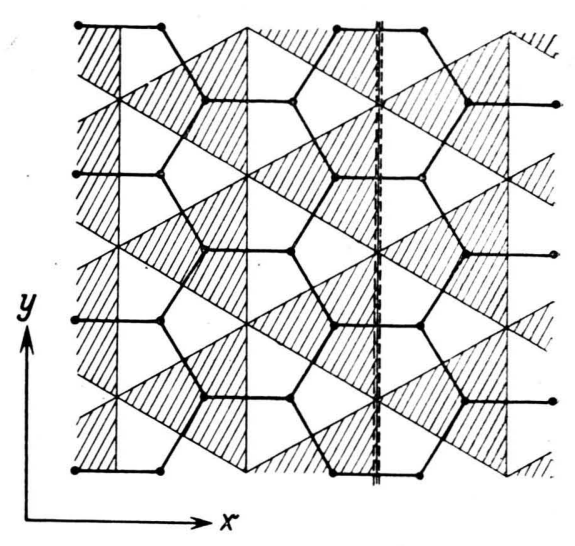

Abb. 7 .

Abb. 5. Die ersten vier Brillouinschen Zonen eines ebenen Rechteckgitters. Im Gültigkeitsbereich der zugrundeliegenden Näherung sind über den Bändern derjenigen Zonen, die nur von ausgezogenen Geraden begrenzt werden, keine Oberflächenzustände zu erwarten.

Abb. 6. Schematische Darstellung eines in der Papierebene liegenden zweidimensionalen Kristalles, dessen Potential ven der Form $V(x)+V(y)$ ist. Die Linien bedeuten Potentialrinnen.

Abb. 7. Zweidimensionaler Kristall der Koordinationszahl 3. Jede Gitterzelle ist noch in eine in der Abb. weiße und schraffierte „Atomzelle“ aufgeteilt.

Zutreffen dieser Näherung oberhalb der Bänder der ersten und zweiten Brillouinschen Zone keine O.Z. auftreten können. Solange als bei dreidimensionalen Gittern, wo die Verhältnisse ähnlich sind, diesen Zonen die Valenzbänder zugeordnet werden dürfen, kann man das Auftreten von O.Z. der besprochenen Art verneinen.

Shockley ${ }^{8}$ hat eine von $\alpha V_{\max }$ unabhängige Extrapolation seines Ergebnisses, das in Abb. 2 für eindimensionale Kristalle dargestellt ist, vorgeschlagen. Wenn man das in die Schrödinger-Gleichung eingehende Potential in der Form

$$
V(x, y, z)=V(x)+V(y)+V(z)
$$

darstellen kann, so läßt sich die dreidimensionale Schrödinger-Gleichung in drei eindimensionale Gleichungen separieren, deren Lösungen bzw. Eigenwerte man nach Abb. 2 schon kennt. Man könnte dann schließen, daß nach dem Kreuzen von Energiebändern dreidimensionaler Kristalle O.Z. auftreten müßten, die zur Hälfte aus dem oberen und zur Hälfte aus dem unteren Band entstammen. $\mathrm{Zu}$ dem Potentialansatz (2) ist jedoch zu bemerken, daß diese Zerlegung nicht nur für das Kristallinnere, sondem vielmehr für den ganzen unendlichen Raum gültig sein muß, wenn die Lösungen den Randbedingungen an der Kristalloberfläche genügen sollen. In Abb. 6 ist gemäß Gl.(2) ein Potential gezeichnet, das einem in der Papierebene liegenden zweidimensionalen Kristall entspricht. Die Linien bedeuten hierbei Potentialrinnen, wobei natürlich zwischen zwei Rinnen ein Berg zu denken ist. Abgesehen von dem unnatürlichen Potentialverlauf im Inneren, entspricht die Oberfläche mit ihren nach dem Unendlichen verlaufenden Potentialrinnen keineswegs realen Kristallen. Es ist deshalb nicht verwunderlich, wenn die gezogenen Folgerungen Widersprüche mit der Erfahrung ergeben. Beim Diamanten haben sich zwei Bänder in dem oben geforderten Sinne überkreuzt $^{17,18}$. Da das obere Band leer und das untere besetzt ist, müßte, wenn Shockley recht hätte, das austretende Oberflächenband halb gefüllt sein und eine Oberflächenleitfähigkeit zur Folge haben.

\section{Rechnung nach der Zellenmethode}

Nach den gemachten Ausführungen können wir bis jetzt bei mehrdimensionalen realen Gittern nur Aussagen im Gültigkeitsbereich der Näherung von freien Elektronen machen. Da es noch keine exakte Lösungsmethode gibt, kommt uns ein Näherungs-

$17 \mathrm{~K}$ i m b a ll, J. chem. Physics 3, 560 [1935].

18 F. H und, Ber. Verh. sächs. Akad. Wiss. 87, 186 [1935]. 
verfahren nach der Zellenmethode ${ }^{19-21}$ sehr gelegen, das nun andererseits mehr von der Seite der gebundenen Elektronen herkommt und sogar bei Gittern mit hohen Koordinationszahlen (d. h. vielen Entwicklungsgliedern) auch im Falle freier Elektronen gute Ergebnisse liefert. In dieser Näherung wurde auch das Kreuzen der Bänder beim Diamant errechnet, und es erscheint uns daher konsequent, in derselben Näherung nach O.Z. zu fragen. Einfachheitshalber wollen wir uns auf das zweidimensionale hexagonale Gitter mit der Koordinationszahl 3 beschränken, das in Abb. 7 dargestellt ist ${ }^{22}$. Es besitzt, wie noch zu zeigen ist, dasselbe Energiespektrum wie der Diamant, und man kann es aus diesen und anderen Gründen mit gutem Recht als zweidimensionales Analogon zum Diamantgitter betrachten. In jeder Gitterzelle befinden sich zwei Atome. Für unsere Rechnung teilen wir jede derartige Gitterzelle noch in zwei „Atomzellen“, von denen wir jeweils die eine schraffieren und die andere weiß lassen. Die Lösung in einer herausgegriffenen Gitterzelle setzen wir dann aus den Teillösungen in den beiden Atomzellen zusammen. Unter der Annahme, daß das Potential in der Umgebung der Kerne radialsymmetrisch ist, machen wir für diese die Ansätze:

$$
\begin{aligned}
& \psi_{\text {weiß }}=A s(r)+2 B p(r) \frac{x}{r}+\frac{2}{\sqrt{3}} C p(r) \frac{y}{r}+\ldots \\
& \psi_{\text {schr. }}=D s(r)+2 F p(r) \frac{x}{r}+\frac{2}{\sqrt{3}} G p(r) \frac{y}{r}+\ldots
\end{aligned}
$$

Die Gln. (3) und (4) können als Anfänge von Entwicklungen nach Cosinus- und Sinusfunktionen (bei dreidimensionalen Problemen entspr. nach Kugelfunktionen) aufgefaßt werden. $s(r)$ und $p(r)$ sind die zugehörigen Radialanteile, die durch numerische Integration aus der Schrödinger-Gleichung zu gewinnen sind. Die Lösung in anderen Gitterzellen geht bekanntlich aus (3) und (4) durch Multiplikation mit $e^{i \mathfrak{t} \mathfrak{r}}$ hervor, wo $\mathfrak{f}$ der sogenannte Ausbreitungsvektor und $\mathfrak{r}$ der Verbindungsvektor entsprechender Punkte der beiden Gitterzellen ist. Damit aber ist die Lösung im Gesamtkristall festgelegt. Die Konstanten $A, B$, $C$ usw. bestimmen sich aus der Forderung, daß die Lösungen benachbarter Atom- bzw. Gitterzellen an den Zellgrenzen stetig ineinander übergehen. Bekanntlich macht man jetzt die Vereinfachung, daß

19 E. Wigner u. F. S e itz, Physic. Rev. 43, 804 [1933].

20 J. C. S l a te r, Physic. Rev. 45, 794 [1934].

21 E. Wigner u. F. S e itz, Physic. Rev. 46, 509 [1934]. man diese Stetigkeitsbedingungen nur in der Mitte der Atom- bzw. Gitterzellgrenzen befriedigt. Dies ergibt in unserem Fall sechs Gleichungen, aus denen wir in (3) und (4) nur je drei Konstanten bestimmen können. Man bricht deshalb die Reihen nach drei Gliedern ab. (Einen ähnlichen Ansatz würde man bei einer Störungsrechnung mit Fastentartung von $s$ - und $p$-Zuständen machen. Man darf wohl erwarten, daß bei nicht zu kleinen Gitterkonstanten die Näherung für Energien von der Größe der der ungestörten $s$ und $p$-Zustände gültig ist.) Aus den Stetigkeitsforderungen an den Zellgrenzen ergibt sich explicite folgendes Gleichungssystem:

$$
\begin{aligned}
A s-2 B p & =D s+2 F p, \\
A s+B p+C p & =(D s-F p-G p) e^{i(\xi+\eta),} \\
A s+B p-C p & =(D s-F p+G p) e^{i(\xi-\eta)}, \\
-A s^{\prime}+2 B p^{\prime} & =D s^{\prime}+2 F p^{\prime}, \\
A s^{\prime}+B p^{\prime}+C p^{\prime} & =\left(-D s^{\prime}+F p^{\prime}+G p^{\prime}\right) e^{i(\xi+\eta)}, \\
A s^{\prime}+B p^{\prime}-C p^{\prime} & =\left(-D s^{\prime}+F p^{\prime}-G p^{\prime}\right) e^{i(\xi-\eta)} .
\end{aligned}
$$

Mit den Abkürzungen

$$
\begin{aligned}
s=s(a / 2) ; \quad p=p(a / 2) ; \quad s^{\prime}=s^{\prime}(a / 2) ; \quad p^{\prime}=p^{\prime}(a / 2) ; \\
\xi=\frac{3}{2} a k_{x} ; \quad \eta=\frac{\sqrt{3}}{2} a k_{y}
\end{aligned}
$$

( $a=$ Abstand zweier Nachbaratome).

Das Verschwinden der Koeffizientendeterminante ergibt die Beziehungen

$$
\begin{gathered}
p p^{\prime}=0, \quad \text { bzw. } \\
\left(\frac{s p^{\prime}+s^{\prime} p}{s p^{\prime}-s^{\prime} p}\right)^{2}+\frac{1}{2} \frac{s s^{\prime} p p^{\prime}}{\left(s p^{\prime}-s^{\prime} p\right)^{2}} \\
=\frac{1}{4}+\frac{1}{2} \cos \xi \cos \eta+{ }_{4}^{1} \cos 2 \eta
\end{gathered}
$$

Für reelles $\xi$ und $\eta$ (dies bedeutet normale Gitterzustände) kann die rechte Seite von $(7 \mathrm{~b})$ nie größer als Eins werden. Die linke Seite ist aber gleich Eins für jeden der vier Fälle

$$
s=0 ; \quad p=0 ; \quad s^{\prime}=0 ; \quad p^{\prime}=0 .
$$

Hierbei ist zu bemerken, daß jede dieser Größen eine Funktion von $a$ und der Energie $E$ ist (weil $E$ in der Schrödinger-Gleichung steht). $s(a / 2, E)=0$

22 F. H und, Ber. Verh. sächs. Akad. Wiss. 87, 325 [1935]. In dieser Arbeit ist gleich zu Beginn beim Ansatz der Gleichungen ein Vorzeichenfehler unterlaufen. Wir konnten deshalb auf die Ergebnisse dieser Arbeit nicht zurückgreifen und haben die Rechnung in unserer Arbeit wiederholt. 
z. B. bedeutet eine Kurve in der $E, a$-Ebene, die ein Gebiet, in dem es noch reelle f-Vektoren, d. h. erlaubte Gitterzustände, gibt, von einem solchen mit nur komplexen $\mathfrak{f}$-Werten trennt. Weitere derartige Grenzkurven existieren nicht, da der kleinste Wert der rechten Seite von $(7 \mathrm{~b})$ für reelles $\mathfrak{f}$ von selbst mit dem kleinsten Wert der linken Seite übereinstimmt. $p=0$ und $p^{\prime}=0$ in (7a) bedeuten in dieser Näherung unaufgespaltene Bänder, da die f́-Abhängigkeit weggefallen ist. Damit ergibt sich das in Abb. 8 gezeichnete Energieschema, cakas, wie oben schon erwähnt, das gleiche Aussehen wie das des Diamanten zeigt ${ }^{23}$. Um O.Z. betrachten zu können, schneiden

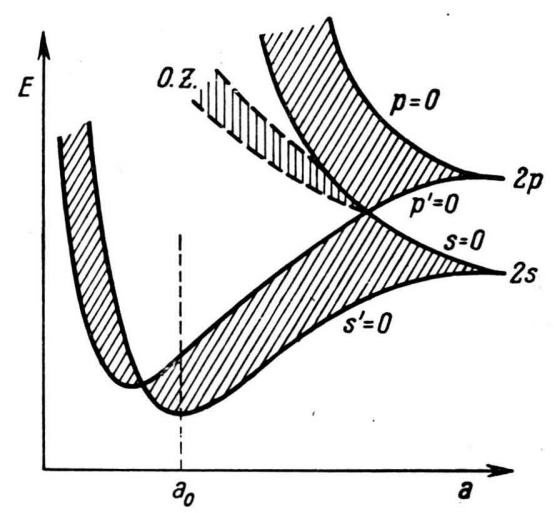

Abb. 8. Energiespektrum als Funktion der Gitterkonstanten des in Abb. 7 dargestellten Kristalles, der längs der dort doppelt gestrichelten Linie durchgeschnitten ist.

wir den Kristall längs der in Abb. 7 doppelt gestrichelten Linie durch und nehmen dabei an, daß er sich an der Oberfläche nicht verzerrt. Aus Symmetriegründen gibt es noch zwei gleichwertige Schnitte. Jetzt müssen wir aber auch Zustände mit komplexem $f$ zulassen, sofern sie nur Funktionen ergeben, die senkrecht zur Oberfläche in den Kristall hinein gedämpft sind. Als Bedingung tritt hinzu, daß die Kristallfunktion sich stetig an eine Vakuumfunktion anschließen muß. Wie es innerhalb der Zellenmethode konsequent ist, verlangen wir den Anschluß nur an einem Punkt jeder Zellenfläche, die in die Oberfläche des Kristalles zu liegen kommt. An einem derartigen Punkt ist nach Abb. 7 und Gl. (4)

$$
\left(\begin{array}{c}
\psi^{\prime} \\
\psi
\end{array}\right)_{\text {Kristall }}=\frac{s^{\prime}+\frac{2 F}{D} p^{\prime}}{s+{ }_{D}^{2 F} p} .
$$

23 Der qualitative Verlauf der Kurven $s:=0, s^{\prime}=0$ usw. ist den Arbeiten von F. H und 18, 22 entnommen.
Wählt man als Vakuumfunktion eine gedämpfte ebene Welle von der Form

$$
e^{-\sqrt{k_{y}{ }^{2}-2 m} \hbar^{2} E x+i k_{y} y}
$$

mit der Energie $E$ der Kristallfunktion, so ergibt sich entsprechend

$$
\left(\frac{\psi^{\prime}}{\psi}\right)_{\text {Vakuum }}=-\sqrt{k_{y}{ }^{2}-\frac{2 m}{\hbar^{2}} E} E
$$

Wir setzen hier im Gegensatz zum Abschn. II im Vakuum $V=0$, also $V_{\text {Kristall }} \neq 0$. Wenn man noch berücksichtigt, daß die beiden tangentialen Ausbreitungsvektoren der Kristall- und Vakuumfunktion einander gleich sein müssen, so lauten die damit zu befriedigenden Anschlußbedingungen:

$$
\begin{aligned}
\left(\psi^{\prime} / \psi\right)_{\text {Kristall }} & =\left(\psi^{\prime} / \psi\right)_{\text {Vakuum }}, \\
k_{y \text { Kristall }} & =k_{y \text { Vakuum }} .
\end{aligned}
$$

$\mathrm{Zu}$ (9) errechnet sich aus dem Gleichungssystem (5)

$\frac{2 F}{D}=\frac{1}{3}\left\{\frac{-2}{\frac{p^{\prime}}{s^{\prime}}+\frac{p}{s}}-\left(\begin{array}{c}s \\ p\end{array}+\frac{s^{\prime}}{p^{\prime}}\right)+\frac{\left(\frac{s}{p}-\frac{s^{\prime}}{p^{\prime}}\right)^{2}}{\frac{s}{p}+\frac{s^{\prime}}{p^{\prime}}} e^{i \xi} \cos \eta\right\}$.

Zu Gl. (12) tritt noch jeweils eine der beiden Determinantenbedingungen (7). Im folgenden betrachten wir nur die Bedingung (7b). Erst am Schluß dieses Abschnittes gehen wir dann kurz auf (7a) ein.

Da die allgemeine Behandlung des Problems immer noch zu kompliziert ist, wollen wir uns weiter darauf beschränken, diejenigen Stellen aufzusuchen, an denen die Oberflächenbänder aus den normalen Bändern austreten. Das Austreten eines Oberflächenbandes ist gleichzeitig ein Kriterium für das Auftreten der O.Z. überhaupt. Es ist offenbar unmöglich, daß ein ganzes Oberflächenband etwa ein ganzes Stück weit in einem normalen Band verläuft und dann erst austritt. Man kann dies aus weiter unten stehenden Beziehungen ersehen. Nach (13) und (16) ist die rechte Seite von $(7 \mathrm{~b})$ z. B. für den O.Z. $\eta=0$ immer größer als Eins. Der Zustand liegt also immer außerhalb eines normalen Bandes. Es gilt jetzt zu untersuchen, wo längs der Grenzkurven $\mathrm{s}=0 ; p=0$; $s^{\prime}=0, p^{\prime}=0$ die Bedingung (11) erfüllbar ist. Wir setzen, indem wir gemäß unserer Verabredung nur O.Z. untersuchen,

$$
\xi=\beta-i \gamma .
$$


Damit wird aus (7b) längs dẹ Grenzkurven

$$
\frac{3}{4}=\frac{1}{2} \cos \eta\left\{\cos \beta \Xi_{0} \gamma \gamma+i \sin \beta \Theta_{i n} \gamma\right\}+\frac{1}{4} \cos 2 \eta \text {. }
$$

$\mathrm{Da}$ das imaginäre Glied verschwinden muß, ist

$$
\beta=0, \pi, 2 \pi, 3 \pi \ldots
$$

Um (14) überhaupt erfüllen zu können, muß weiter sein

a) für $\cos \eta>0: \beta=0,2 . \tau, 4 . \pi \ldots$

b) für $\cos \eta<0: \beta=\pi, 3 \pi, 5 \pi \ldots$

Gl. (16) gilt auch für O.Z., die außerhalb der Bänder liegen, da dort die linke Seite von (14), wie leicht $\mathrm{zu}$ zeigen ist, nur größer sein kann. Der Ausdruck $e^{i \xi} \cos \eta=e^{i \beta} e^{\gamma \gamma} \cos \eta$ in Gl. (12) ist also nach (16) stets positiv. Ersetzt man noch $\mathcal{E v o j}_{\gamma}$ durch $e^{\gamma}$, was für die folgenden Abschätzungen meist genügt, so wird gemäß (14)

$$
e^{i \beta} e^{\gamma} \cos \eta=|\cos \eta| e^{\gamma} \approx \frac{3-\cos 2 \eta}{2} .
$$

[genauer gesagt kann $|\cos \eta| e^{r}$ höchstens den doppelten, durch (17) gegebenen Wert annehmen].

Wir beginnen nun mit der Untersuchung, wo die Anschlußbedingung (11) längs der Grenzkurven in dem Energiespektrum der Abb. 8 erfüllbar ist, d. h. wir suchen die Stellen, wo die Oberflächenbänder austreten.

a) Längs der Grenzkurve $p=0$ :

Setzt man (12) in (9) ein, so ergibt sich unter Berücksichtigung von (17) mit $p=0$

$$
\left(\psi^{\prime} / \psi\right)_{\text {Kristall }}=\infty \text {. }
$$

Ein Anschluß an (10) gemäß der Bedingung (11) ist also sicher nicht möglich.

b) Längs der Grenzkurve $p^{\prime}=0$ :

Hier ergibt sich entsprechend

$$
\left(\psi^{\prime} / \psi\right)_{\text {Kristall }}=0 .
$$

Ein Anschluß ist hier ebenfalls nicht möglich.

c) Längs der Grenzkurve $s=0$ :

$$
\left(\frac{\psi^{\prime}}{\psi}\right)_{\text {Kristall }}=\begin{gathered}
p^{\prime} \\
p-1+|\cos \eta| e^{\gamma} \\
-1+|\cos \eta| e^{\gamma}
\end{gathered} .
$$

In (20) ist der Faktor von $p^{\prime} / p$ wegen (17) stets positiv. Ein Anschluß an (10) wird erst dort möglich, wo $p^{\prime} / p$ negativ ist. $p^{\prime} / p$ (längs $s=0$ ) ändert dort sein Zeichen, wo sich die Kurven $s=0$ und $p^{\prime}=0$ schneiden. Aus den graphischen Lösungen ${ }^{18}$ für $p$ und $p^{\prime}$ entnimmt man, daß der Quotient $p^{\prime} / p$ links von der Kreuzungsstelle negativ wird. In Umgebung dieser Stelle ist zwar $p^{\prime} / p$ sehr klein, aber der Faktor $\left(2+|\cos \eta| e^{\gamma}\right) /\left(-1+|\cos \eta| e^{\gamma}\right)$ kann für $|\cos \eta| e^{r} \approx 1$ sehr große Werte annehmen. Wie man sich genau überlegt, ist die Anschlußbedingung für sämtliche Werte von $k_{y}$ von der Kreuzungsstelle an längs dieser Grenzkurve in einem ganzen Bereich von $p^{\prime} / p$ erfüllbar. In Abb. 8 ist dieser Bereich durch ein austretendes Oberflächenband qualitativ angedeutet (gestrichelt gezeichnet).

d) Längs der Grenzkurve $s^{\prime}=0$ :

Hier ergibt sich

$$
\left(\frac{\psi^{\prime}}{\psi}\right)_{\text {Kristall }}=\frac{p^{\prime}-1+|\cos \eta| e^{\gamma}}{p} \frac{2+|\cos \eta| e^{\gamma}}{} .
$$

In diesem Ausdruck liegen die Verhältnisse ähnlich wie in (20). $p^{\prime} / p$ erhält erst dort das richtige Vorzeichen, wo sich die Grenzkurven $p^{\prime}=0$ und $s^{\prime}=0$ schneiden. In unmittelbarer Nähe dieses Schnittpunktes ist aber ein Anschluß wie in (20) nicht möglich, da der Faktor $\frac{-1+|\cos \eta| e^{\gamma}}{2+|\cos \eta| e^{\gamma^{\prime}}}$ keine sehr großen Werte annehmen kann. Wir müssen aber bemerken, daß man bei so kleinen Werten von $a$ wohl schon mehr Entwicklungsglieder in Gl. (3) und (4) brauchen wird und deshalb dieses Ergebnis unsicher ist. Diese Unsicherheit wirkt sich jedoch beim Vergleich mit der Erfahrung nicht störend aus, da diese Werte von $a$ beim Diamanten bereits jenseits der realen Gitterkonstanten $a_{0}$ liegen.

Es bliebe jetzt noch die Bedingung ( $7 \mathrm{a}$ ) zu untersuchen. Sie ergibt, wie man sieht, überhaupt keine Lösungen außerhalb der beiden zu ihr gehörigen unaufgespaltenen „Bänder“, d. h. auch keine O.Z. Austretende O.Z., die wir oben nur untersucht haben, gibt es also nicht. Man kann hier noch weiter gehen und zeigen, daß auch innerhalb der beiden „Bänder“ die Anschlußbedingung (11) im allgemeinen nicht befriedigt werden kann. Doch soll dies nicht näher ausgeführt werden.

Die Zellenmethode ergibt also, daß die an der Kreuzungsstelle austretenden O.Z. nur aus dem oberen Band entstammen. Man hat damit ein Beispiel gefunden, das direkt zeigt, daß die Extrapolation vom eindimensionalen aufs mehrdimensionale Gitter nicht statthaft ist.

Die Anregung zu dieser Arbeit sowie zahlreiche wertvolle Ratschläge und Diskussionen verdankt der Verfasser Hrn. Prof. Dr. E. F u e s. 